\title{
Contingencias en salud y Medicina Centrada en la Persona
}

\author{
Nelson Raúl Morales Soto ${ }^{l}$
}

Los desastres ocasionan mortalidad y destrucción con impacto económico y social creciente. Por ello y por sus efectos en la salud individual y colectiva son de interés en medicina y salud pública. Ninguna comunidad puede sentirse exceptuada del riesgo de desastres de origen en la naturaleza o asociados a las actividades del hombre. El enfrentamiento al problema requiere intervención multisectorial siendo de gran interés la salud y la educación. Se prevé incremento de efectos y de daños por el cambio climático y el deterioro de las relaciones humanas.

En los veinte últimos años del siglo XX tres millones de personas murieron por efecto de desastres naturales ${ }^{(1)}$, en 2010 fallecieron 296,800 personas y otros 208 millones sufrieron lesiones por 373 desastres naturales que costaron $110 \mathrm{mil}$ millones de dólares; terremotos en América Latina concentraron el $75 \%$ de la mortalidad -Haití- y 30\% de las pérdidas mundiales -Chile-(2), 10 a $15 \%$ de las víctimas desarrolla limitaciones físicas o mentales crónicas ${ }^{(3)}$.

Toda comunidad ha desarrollado mecanismos de protección y respuesta a desastres, tienen mejor preparación aquellas que sufren impactos periódicos (huracanes, grandes lluvias). El Perú ha sido escenario de desastres de variado origen. En Lima, capital del país, la Asistencia Pública de Lima concentró en su red de servicios la atención de urgencias y emergencias a la comunidad hasta que su local central se trasladó a lo que hoy es el Hospital Nacional Casimiro Ulloa al iniciarse la década de los 80 . Ese fue el centro de entrenamiento del personal de emergencia en su forma no escolarizada.

En 1965 se aprobó el primer Plan Hospitalario para Desastres en el Hospital Central de Policía de Lima. Fue probado y validado en los terremotos de 1966, 1970 y 1974.

En 1980, al iniciarse la guerra contra el terrorismo, el Hospital de Policía toma la iniciativa de implementar en su servicio de emergencia medidas para la atención del paciente con gravedad súbita extrema (visión indispensable para víctimas por armas de fuego y explosivos) instalando un área de Triage y una Unidad de Shock-Trauma, las primeras en el país, cirugía del trauma, transporte asistido del paciente grave en ambulancias equipadas y con radio comunicación. Se formó el primer equipo de médicos y enfermeros con un modelo escolarizado que permitió luego la formación escolarizada de especialistas la cual se inició en 1993 en la Universidad Nacional Mayor de San Marcos ${ }^{(4)}$.
En el plan curricular del año 2008 se incorporó el curso "Ética, derechos humanos y aspectos legales del ejercicio médico" ("Curso Dr. Pedro Ortiz Cabanillas"). Desde el año 2000 hasta 2013 se dictó un curso para ingresantes al pregrado de medicina sobre este tema. Entre los años 2014 y 2015 se fue incorporando el concepto de Medicina Centrada en las Personas en los cursos de pre y post grado de la especialidad. Esto ha enriquecido notablemente la comprensión de conceptos humanistas y éticos y su utilización en la atención del paciente de emergencia.

Toda persona que acude buscando atención en servicios de emergencia tiene algún grado de aprensión. Cuando hay desmedro rápido de las funciones vitales básicas (respiración, circulación, hidratación, metabolismo, estado de conciencia) la ansiedad es grande en el paciente y en sus familiares, más aún cuando el origen de los daños es ocasionado por traumatismos, accidentes, violencias o desastres.

El personal médico requiere criterios claros para afrontar y atender exitosamente estas situaciones que alcanzan la atención de los medios masivos cuando se trata de víctimas en masa o cuando median factores delictivos o conflictos sociales. El Emergenciólogo requiere no solo adecuadas competencias cognitivas, operativas y éticas en su formación especializada, sino también debe tener una clara comprensión del fenómeno social para medir el impacto que su opinión comunicación al familiar y al público- tiene en la comunidad. La Medicina Centrada en las Personas es un gran aporte a este propósito.

En años recientes se ha acrecentado el esfuerzo en la implementación de la Gestión del Riesgo de Desastres y se han creado organismos oficiales con este encargo legal (Sistema Nacional de Gestión del Riesgo de Desastres, SINAGERD) lo cual ha potenciado al Sistema Nacional de Defensa Civil. Uno de sus objetivos principales es crear una cultura de la prevención y la resiliencia al evento adverso.

El sistema de salud debe garantizar la atención resolutiva del paciente en situación de emergencia en la cadena de servicios de emergencia de los hospitales del Sector Salud asegurando los recursos según el nivel tecnológico del establecimiento.

Son vacíos por completar la creación de un Sistema Nacional de Atención Médica de Emergencias y Desastres que

${ }^{\prime}$ Academia Nacional de Medicina. Universidad Nacional Mayor de San Marcos (UNMSM). Sociedad Peruana de Medicina de Emergencias y Desastres. 
cuente con centrales de referencia del paciente grave en las grandes ciudades, de un fondo permanente que permita la atención del grave en las horas críticas en cualquier establecimiento y la educación transversal en la reducción de la vulnerabilidad en toda la población.

Debemos tener presente que las contingencias de salud tienen un alto costo biológico, económico y social para las víctimas y el país. Las más frecuentes -urgencias y emergencias cotidianas- afectan la salud, la vida y la economía empobreciendo familias y comunidades ${ }^{(5-9)}$ predisponiéndolas ${ }^{(10-11)}$ para ser víctimas de eventos extremos: los desastres de origen natural o antrópico. Lima concentra la mayor incidencia y peligrosidad de tales siniestros los cuales, por otro lado, han impulsado el desarrollo de los servicios de emergencia y la institucionalización para afrontar eventos catastróficos).
Lima, la ciudad más poblada del país, ha sido afectada por terremotos destructores cada siglo. Estudios técnicos del Instituto Nacional de Defensa Civil, INDECI ${ }^{(12)}$, analizan la probable ocurrencia de un terremoto, seguido de maremoto, en el litoral central del Perú, cuya magnitud podría alcanzar los 8 grados Richter; los expertos estiman que 200 mil viviendas quedarían destruidas y 348 mil inhabitables, esto ocasionaría unas 51 mil muertes y entre 50 mil a 686 mil heridos en Lima y el Callao. Esta región registra históricamente un gran terremoto cada siglo, la zona en riesgo -litoral y sierra central- se extiende a 250 mil km2 y en ella viven 14 millones de habitantes, algunos en áreas geográficas de difícil acceso ${ }^{(13)}$.

Debe ampliarse la aplicación de la Medicina Centrada en las Personas en las contingencias de salud como una vía que permita la aplicación de las propuestas.

\section{Referencias bibliográficas}

1. Noji E. Editor. 2000. Impacto de los Desastres en la salud pública. Editorial Linotipia Bolívar Ltda. Bogotá, 2000.

2. Organización Panamericana de Salud, OPS/OMS. Centro de Investigación sobre Epidemiología de los Desastres, CRED, 2011. http://www.paho.org/disasters/newsletter/index.php? option $=$ com content $\&$ view $=$ article $\&$ id $=456 \% 3$ Acredprovides-valuable-information-on-the-impact-ofdisasters \& catid $=214 \% 3$ A issue-115-april-2011-newtools\&Itemid=285\&lang=es, acceso 14 enero2015.

3. Cohen Raquel.hhttp://www.raquelcohendisaster.com/

4. Universidad Nacional Mayor de San Marcos. Plan curricular de Medicina de Emergencias y Desastres. 2008.

5.INDECI. Emergencias a nivel nacional. 2010. anualhttp:/www.indeci.gob.pe/objetos/secciones/MTM=/NT $\mathrm{M}=/ \mathrm{lista} / \mathrm{NTY} 5 / 201106161210221 . \mathrm{pdf}$, acceso 30octubre2012.

6. Ministerio de Salud. Estrategia Sanitaria Nacional de Accidentes de Tránsito. www.minsa.gob.pe/ogdn/esp/pdf/ Plan\%20General\%20Accidentes\%20de\%20Transito, acceso 28 de mayo 2010.

7. Ministerio de Salud. Políticas municipales para la promoción de la seguridad vial. Lima: OPS; 2005.

8.INEI. Estadísticas de seguridad ciudadana. No 2. Feb 2012.
http://economia.unmsm.edu.pe/Servicios/BEst/datos/INEI IT 24.2.12_ESC.pdf, acceso 31 octubre2012

9. Villanueva R., y col. Homicidio y feminicidio en el Perú. Setiembre 2008-Junio 2009. Ministerio acceso 30octubre2012.

10. Yamada G. 2001. Reducción de la pobreza y fortalecimiento del capital social y la participación: La acción reciente del Banco Interamericano de Desarrollo. Conferencia Regional "Capital Social y Pobreza", CEPAL, Santiago de Chile. http://www.iadb.org/sds/doc/POVRPFCS.pdf acceso 08oct09.

11. Morales-Soto, NR. Desastres: La construcción de la vulnerabilidad. Anales de la Acad Nac Med: 2009;65-78. http://www.acadnacmedicina.org.pe/publicaciones/Anales $\% 2$ 02009/sesion_extraordinaria_incorporacion_academico_Nels on_Raul_Morales.pdf

12. INDECI/PREDES. 2009. Diseño de Escenario sobre el Impacto de un Sismo de Gran Magnitud en Lima Metropolitana y Callao, Perú. http://www.indeci.gob.pe/plan_a_sismo/d esc sis lima.pdf, acceso 12abril2012

13. Morales-Soto N, Zavala C. Terremotos en el Litoral Central del Perú: ¿Podría ser Lima el escenario de un futuro desastre?. Rev Peru Med Exp Salud Pública. 2008;25(2):217-224. http://www.scielo.org.pe/pdf/rins/v25n2/a11v25n2.pdf 